\title{
CAPACIDADES Y LEYES FENOMENOLÓGICAS: EL DISPOSICIONALISMO EXPERIMENTAL ${ }^{1}$
}

\author{
Cristian Soto \\ Departamento de Filosofía \\ Universidad de Chile \\ cssotto@gmail.com \\ Pascal Rodríguez \\ Departamento de Filosofía \\ Universidad de Chile \\ pascal.rodriguez@ug.uchile.cl
}

\begin{abstract}
RESUMEN / ABSTRACT
Elaboramos una versión del disposicionalismo experimental que esclarece la relación entre capacidades y leyes fenomenológicas. Tras algunas consideraciones introductorias (1), presentamos el disposicionalismo experimental en vistas de las nociones de modalidad física y ambiente de estímulo (2). Trazamos las líneas generales para una epistemología experimentalista de las capacidades (3) y esclarecemos la modalidad física e invariancia de las leyes fenomenológicas (4). Luego examinamos las generalizaciones físicas y extrapolaciones de las leyes fenomenológicas (5) y exponemos el caso de la ley de Fresnel, que ilustra aspectos de nuestro argumento (6). Concluimos con algunas consideraciones sobre los límites del disposicionalismo experimental.
\end{abstract}

Palabras Clave: disposicionalismo experimental, capacidades, leyes fenomenológicas, modalidad física, invariancia, extrapolación.

\section{CAPACITIES AND PHENOMENOLOGICAL LAWS: FOR EXPERIMENTAL DISPOSICIONTALISM}

We elaborate a form of experimental dispositionalism that illuminates the relationship between capacities and phenomenological laws. After some introductory remarks (1), we introduce experimental dispositionalism in view of the notions of physical modality and environment of stimulus (2). Such notions are crucial for both outlining an experimental epistemology for capacities (3) and understanding the physical modality and invariance of phenomenological laws (4). We characterize physical generalizations and extrapolations licensed by phenomenological laws (5), and examine the Fresnel law, which illustrates key elements of our argument (6). We close with remarks on the scope and limits of experimental dispositionalism (7).

KEYWORDS: Experimental dispositionalism, capacities, phenomenological laws, physical modality, invariance, projection.

$1 \quad$ El siguiente artículo es resultado del FONDECYT de Iniciación No 11160324 "The PhysicoMathematical Structure of Physical Laws". Investigador responsable: Dr. Cristian Soto. 


\section{Sobre leyes y capacidades}

R⿴囗十 Discusiones recientes en filosofía de las ciencias han arrojado luz sobre la relación entre leyes de la naturaleza-leyes cientificas o simplemente leyes-y capacidades. Un problema, a este respecto, consiste en examinar la prioridad ontológica que podría establecerse entre leyes y capacidades. Por un lado, algunos sostienen que las leyes tienen prioridad ontológica respecto de las capacidades, a saber: uno de los roles de las leyes consistiría en gobernar o determinar las relaciones estructurales que tienen lugar entre las distintas capacidades que instancian fenómenos particulares. El objetivo de las ciencias físicas consistiría en el descubrimiento de tales leyes, las cuales facilitarían las tareas de construir descripciones, explicaciones y predicciones de fenómenos según las leyes que gobiernen sus capacidades.

Por otro lado, contamos con diversas elaboraciones de la tesis que sostiene que las capacidades tienen prioridad ontológica respecto de las leyes. Según ello, los fenómenos tendrían lugar en virtud de las capacidades que poseen, otorgándoles un conjunto de poderes causales que posibilitan interacciones físicas específicas. Las capacidades serían propiedades sensibles al ambiente que confieren tendencias a comportarse de una manera u otra a los objetos que las poseen (Cartwright 1989). Las leyes, en este contexto, estarían determinadas por el conjunto de capacidades que posean los fenómenos.

Recuérdese la distinción de Cartwright (1983, p. 1) entre leyes fundamentales y leyes fenomenológicas. Las leyes fundamentales pretenden explicar aspectos ubicuos de la realidad, a pesar de no ser verdaderas si se las considera descripciones que enuncian hechos. Empleando diversos tipos de abstracciones e idealizaciones de alto nivel, las leyes fundamentales se limitan a explicar aspectos del funcionamiento de diversos sistemas ideales instanciados por otros tantos modelos científicos. En cambio, las leyes fenomenológicas describen regularidades que tienen (o pueden tener) lugar en fenómenos específicos que ocupan una región restringida en la historia del universo. La verdad de las leyes fenomenológicas radica en que se trata de enunciados que describen fenómenos a los que se tiene acceso experimental a través de mediciones y detecciones de diversa índole. Las leyes fenomenológicas apelan a abstracciones e idealizaciones de menor alcance, permitiendo referir a fenómenos específicos de manera más intuitiva.

En adelante, enfocaremos nuestro análisis en la relación entre leyes fenomenológicas y capacidades, dejando a un lado la noción de leyes fundamentales. En particular, argumentaremos que el disposicionalismo experimental permite dar cuenta de aspectos clave de la relación entre capacidades y leyes fenomenológicas, sosteniendo que las capacidades son propiedades causales cuya modalidad física determina nuestros enunciados de leyes fenomenológicas. La estructura de nuestro argumento es la siguiente. En la sección 2 argumentaremos que el disposicionalismo experimental revela aspectos cruciales de las capacidades, tales como su modalidad intrínseca y su sensibilidad a ambientes de estímulo. La sección 3 ofrece las líneas generales para una epistemología experimentalista que pueda ser aplicada a las capacidades. La sección 4 muestra que las leyes fenomenológicas describen interacciones entre capacidades, destacando 
que tales leyes derivan su modalidad física e invariancia a partir de las capacidades. La sección 5 examina el carácter de las generalizaciones físicas ofrecidas por leyes fenomenológicas y la posibilidad de extrapolar enunciados de leyes fenomenológicas desde un ambiente experimental a otros. En la sección 6 exponemos el caso de estudio de la ley de Fresnel, que nos permite ilustrar las ideas principales de nuestro argumento. Finalmente, la sección 7 cierra con consideraciones sobre el alcance y los límites del disposicionalismo experimental.

\section{Disposicionalismo experimental: modalidad física y ambiente de estímulo}

Las capacidades son poderes causales que confieren tendencias a comportarse de ciertas maneras a los fenómenos que las poseen. El comportamiento al que las capacidades dan lugar no está determinado de manera única, sino que puede variar según los factores ambientales que tienen lugar en el espacio en el que se manifiestan. Ahora bien, las capacidades podrían no manifestarse en ambientes que no incluyen condiciones de estímulo adecuadas o cuando la manifestación es inhibida por la presencia de capacidades inhibitorias.

El disposicionalismo experimental nos permite dar cuenta de la modalidad física intrínseca de estas propiedades. Introducimos la jerga modal, en este punto, puesto que el conocimiento de las capacidades nos informa no solamente acerca de lo que es el caso, sino acerca de un rango de fenómenos posibles que podrían tener lugar dado un conjunto de capacidades en ambientes adecuados de estímulo.

Mientras que Cartwright (1989) ha empleado la expresión capacidad, otros (Mumford 1998 y Chakravartty 2017) llaman a estas propiedades disposiciones. Nosotros seguimos la primera nomenclatura. Ahora bien, dentro de la misma propuesta de Cartwright, las diferencias entre capacidades y disposiciones varían y generan ambigüedad. Sin ir más lejos, Cartwirght (1999) declara explícitamente que las capacidades son distintas a las disposiciones, mientras que Cartwright (2002) sostiene que son lo mismo. En vistas de ello, reconocemos que su ontología es innecesariamente confusa, coincidiendo con que "es necesario decir más acerca de las capacidades antes de comenzar a pensar seriamente en que se debe tener un compromiso ontológico con ellas" (Psillos 2008, p. 188).

La relevancia del disposicionalismo experimental resulta evidente si se lo compara con concepciones metafísicas estándares del categoricalismo y del disposicionalismo (Mumford 1998, 2009; Chakravartty 2007). Esta distinción entre propiedades categóricas y propiedades disposicionales ha dominado el debate. El siguiente pasaje ofrece una definición estándar de las primeras:

las propiedades categóricas (...) son descritas en términos de aspectos estáticos de particulares tales como sus dimensiones (extensión, volumen), formas (rectangular, cilíndrica), configuraciones de arreglos (estructuras moleculares), y así sucesivamente (Chakravartty 2007, p. 124). 
Por otro lado, la concepción disposicional sostiene que las propiedades confieren capacidades para comportarse de una u otra manera a los objetos que las poseen. Introduzcamos una distinción entre dos formas de disposicionalismo, a saber, el disposicionalismo metafísico estándar (Chakravartty 2007, 2017), y el disposicionalismo experimental. En particular, el disposicionalismo metafísico estándar asocia una capacidad con un único comportamiento:

La idea de que las propiedades causales son multifacéticas no debiera confundirse con la idea de que las propiedades confieren disposiciones diferentes en circunstancias diferentes (...) En términos metafisicos, las propiedades causales son identificadas de manera única en todas las circunstancias con las mismas disposiciones (Chakravartty 2007, pp. 149-50. Énfasis nuestro).

El disposicionalismo experimental se distancia del disposicionalismo metafísico estándar, sosteniendo que las disposiciones les otorgan a los fenómenos que las poseen poderes causales para dar lugar a un rango modal de manifestaciones. Según esto, una capacidad no está asociada a una única manifestación, sino a un abanico de posibles comportamientos. La manifestación que tiene lugar en cada caso particular depende de su relación con otras tantas propiedades disposicionales con las que interactúa en un ambiente de estímulo específico.

El disposicionalismo experimental reconoce la sensibilidad ambiental de las capacidades. La manifestación de las capacidades no es binaria, vale decir, no es una cuestión de si se manifiesta o no. Por el contrario, se pueden generar cambios graduales en las manifestaciones de las capacidades dependiendo de la configuración del ambiente de estímulo, existiendo un continuum experimental entre la manifestación cabal de una capacidad y su no manifestación.

Entendemos que un ambiente de estímulo es cualquier ambiente que resulta propicio para la manifestación de una capacidad. Encontramos al menos dos escenarios distintos de estímulo. Primero, un ambiente de estímulo óptimo es aquel en el que se aprecia la manifestación cabal de una capacidad. Y segundo, un ambiente de estímulo negativo es aquel en el que la presencia de factores identificables inhibe activamente la manifestación de una capacidad.

El concepto de ambiente óptimo -que Cartwright (1999) llama ideal-tiene dos acepciones:

La clave del presente concepto es el concepto ideal [u óptimo]. Por un lado, empleamos este término para señalar el hecho de que las circunstancias en cuestión no son reales o, al menos, que ellas raramente se obtienen de manera natural, sino que requieren de diversos artilugios incluso para aproximarse a ellas. Por otro lado, las circunstancias ideales son las circunstancias 'correctas' -correctas para inferir cuál es la naturaleza de la conducta misma (Cartwright 1999, p. 84).

El ambiente óptimo usualmente se crea artificialmente en la práctica científica con el propósito de facilitar la manifestación cabal de una capacidad en la experimentación. Parte importante de la investigación científica consiste en el diseño y construcción de 
diversos ambientes experimentales óptimos que garanticen la manifestación de una capacidad para, entre otras cosas, permitir su detección y medición. Volveremos sobre este punto a lo largo de nuestro argumento.

Por lo pronto, además de distanciarse del categoricalismo y del disposicionalismo metafísico estándar, el disposicionalismo experimental se opone a la concepción humeana que sugiere que los fenómenos se reducen a constituyentes estáticos inertes (powerless). La modalidad física intrínseca de las capacidades consiste en que estas les otorgan a los fenómenos que las poseen un rango de comportamientos que podrían tener lugar dependiendo del ambiente de estímulo en el que se encuentren. El comportamiento ocurrente es solamente uno de los comportamientos posibles a los que una capacidad puede dar lugar. Las capacidades involucran la posibilidad modal de otros comportamientos que pueden tener lugar en otros escenarios. Uno de los beneficios de esta caracterización de la modalidad física de las capacidades dice relación con que ella acomoda la práctica científica, la cual no se restringe a ofrecer un inventario de lo que es el caso, sino que ofrece información modal acerca de escenarios que tenemos evidencia para creer que podrían haber sido o podrían ser el caso (véase secciones 4 y 5 , en donde aplicamos esta consideración a la noción de ley).

Los fenómenos están constituidos por capacidades y acarrean sus poderes causales de un ambiente a otro. Aunque la manifestación de una capacidad es potencial, la realidad de las capacidades no lo es. Las capacidades pueden o no manifestar sus poderes causales e influir en el comportamiento de los fenómenos. Sin embargo, si un fenómeno no manifiesta una determinada capacidad en un ambiente específico, ello no indica que la capacidad no exista, sino solo que el ambiente no es de estímulo o que quizás este involucra factores inhibitorios. Igualmente, que un fenómeno tenga una manifestación distinta en un ambiente nuevo no significa que el fenómeno haya adquirido una nueva capacidad, sino que los demás ambientes no le eran de estímulo a este respecto. En la sección siguiente desarrollaremos este punto, defendiendo que el criterio de individuación de capacidades en vistas de la adopción de compromisos ontológicos se encuentra en la experimentación.

\section{Una epistemología experimentalista para las capacidades}

La interacción entre capacidades y ambientes de estímulo determina la probabilidad de que se manifieste un poder causal asociado a una capacidad. Una primera aproximación a la causalidad probabilística sostiene que "la causa tiene que elevar la probabilidad del efecto; [...] una instancia del tipo que se asume que es la causa tiene que aumentar la probabilidad de que ocurra una instancia del efecto tipo" (Dupré 1984, p. 169).

Sin embargo, dada la naturaleza de las capacidades, una causa no se caracteriza tan solo por elevar las probabilidades de que tenga lugar el efecto. En rigor, las causas alteran las probabilidades de que ocurra el efecto, pudiendo elevarlas o disminuirlas. Mientras que la definición de Dupré no permite dar cuenta de las capacidades inhibitorias, las cuales se caracterizan por disminuir las probabilidades de que tenga lugar un efecto, 
en lo que sigue asumiremos que una causa puede tanto elevar como disminuir las probabilidades de un efecto.

Concebir la causalidad en términos probabilísticos tiene al menos dos beneficios. Primero, en la práctica científica se recurre a las probabilidades como herramienta para medir influencias causales y para identificar la presencia de propiedades de fenómenos. Y segundo, el análisis probabilístico puede acomodar la modalidad física de la causalidad, facilitando el análisis de la posibilidad de que tenga lugar un proceso causal particular en un ambiente determinado en términos de probabilidad.

En breve, una capacidad se individúa por medio de la articulación de un enunciado condicional que explicita las circunstancias que tienen que darse para que ciertas manifestaciones tengan lugar con determinada probabilidad. Podemos expresar esto en los siguientes términos:

$$
\mathrm{C} \rightarrow(\mathrm{S} \rightarrow \mathrm{M}) \text {, ceteris paribus, } \mathrm{S}=\{\mathrm{S} 1, \mathrm{~S} 2, \mathrm{~S} 3, \ldots, \mathrm{Sn}\} \text { y } \mathrm{M}=\{\mathrm{M} 1, \mathrm{M} 2, \mathrm{M} 3, \ldots, \mathrm{Mn}\}
$$

en donde $\mathrm{C}$ es la capacidad, $\mathrm{S}$ es el conjunto de condiciones experimentales de estímulo, y $\mathrm{M}$ es el conjunto correspondiente de manifestaciones. Este enunciado declara que, si es el caso que $\mathrm{C}$ y $\mathrm{S}$, entonces $\mathrm{M}$ ocurrirá con determinada probabilidad. El condicional debe ser leído como un condicional probabilístico: "Si a es un evento tal que $\mathrm{P}(\mathrm{a})>0$, la probabilidad condicional del evento B, dado A, se define así: $\mathrm{P}(\mathrm{a} / \mathrm{b}) \frac{P(a b)}{\bar{P}(a)}$ " (Mosterín y Torretti 2002, p. 468).

El ambiente de estímulo óptimo es particularmente relevante para la práctica científica, permitiéndonos conocer la manifestación cabal de las capacidades en tanto que garantiza que estas producirán sus efectos sin que la probabilidad sea alterada por otras capacidades. En contraste, la complejidad de ambientes naturales no controlados oscurece nuestro discernimiento epistémico acerca de la incidencia que otras capacidades podrían tener en la manifestación de poderes causales que son objeto de interés en escenarios específicos. Las capacidades se presentan en tramas complejas, que solo la implementación de prácticas y tecnologías sofisticadas de experimentación permiten desenmarañar. Por ejemplo, no sabemos cómo se comporta un electrón tan solo en virtud de su carga eléctrica negativa si nos limitamos a examinar ambientes naturales no controlados. La carga eléctrica negativa de los electrones interactúa con otras capacidades, tales como tener masa y spin, actuando además bajo la influencia de otras diversas fuerzas de la naturaleza. Solamente el diseño de ambientes experimentales artificiales y controlados puede garantizarnos el acceso epistémico a la manifestación de sus poderes causales en escenarios de investigación.

Conocer la manifestación cabal de una capacidad en circunstancias experimentalmente controladas nos permite llevar a cabo generalizaciones empíricas de diverso alcance. La generalización empírica no se restringe al acto de derivar inductivamente un enunciado general a partir de un conjunto iterativo de instancias particulares. Por el contrario, la generalización empírica descansa en la posibilidad modal de extrapolar el conocimiento de la manifestación del poder causal de una capacidad desde un ambiente experimental a otros, incluyendo ambientes naturales en los que la misma capacidad tiene lugar en 
circunstancias similares. Por supuesto, los ambientes naturales no controlados presentan un desafío para la extrapolación de inferencias causales probabilísticas que se obtienen en ambientes experimentales controlados, tal como lo indicaremos en las secciones 4 y 5 en relación con las leyes fenomenológicas.

Concentrémonos en el análisis probabilístico de las capacidades desde la perspectiva del disposicionalismo experimental. El acceso epistémico a las capacidades y el compromiso ontológico con su realidad dependen de nuestra habilidad para diseñar e implementar ambientes experimentales que provean las condiciones de estímulo adecuadas. Interpretado objetivamente, el análisis probabilístico ofrece una herramienta que permite medir influencias causales (Cartwright 1989) y dar cuenta de relaciones físicas modales. En efecto, una causa altera la probabilidad de que ocurra su efecto. Un fenómeno es causa de otro si este último es probabilísticamente dependiente del primero. Tal dependencia se puede ilustrar a través de alguna de las siguientes relaciones:

$$
\begin{aligned}
& \mathrm{P}(\mathrm{e} / \mathrm{c})>\mathrm{P}(\mathrm{e}) \\
& \mathrm{P}(\mathrm{e} / \mathrm{c})<\mathrm{P}(\mathrm{e}) \\
& \mathrm{P}(\mathrm{c} / \mathrm{e})>\mathrm{P}(\mathrm{c}) ; \\
& \mathrm{P}(\mathrm{c} / \mathrm{e})<\mathrm{P}(\mathrm{c})
\end{aligned}
$$

Nótese que la relación es simétrica, vale decir, el hecho de que c y e sean probabilísticamente dependientes no nos informa acerca de si c es causa de e o si e es causa de c. Para elucidar esto se precisa información física adicional proveída por la manipulación en ambientes controlados. Por lo demás, esto significa que c es causa de e (o viceversa) cuando la probabilidad de que ocurra e cuando ha ocurrido c es mayor que cuando no ocurre c (o viceversa). Por el contrario, un fenómeno no es causa de otro si la probabilidad de que ocurra uno se mantiene igual dada la ocurrencia del otro, vale decir, si

$$
\begin{aligned}
& \mathrm{P}(\mathrm{e} / \mathrm{c})=\mathrm{P}(\mathrm{e}) \\
& \mathrm{P}(\mathrm{c} / \mathrm{e})=\mathrm{P}(\mathrm{c})
\end{aligned}
$$

El análisis probabilístico ofrece diversos beneficios para el disposicionalismo experimental, permitiéndonos detectar y adscribir capacidades, otorgándole contenido empírico a la individuación de una capacidad. En efecto, correlacionamos el poder causal de una capacidad con un efecto en un ambiente de estímulo (C, S y M en la formulación de la definición propuesta $)^{2}$. Sin embargo, el análisis probabilístico no nos entrega

2 Uno de los límites del análisis probabilístico de las capacidades consiste en que las probabilidades por sí solas no nos permiten detectar correlaciones espurias o la presencia de procesos causales que escapen al análisis probabilístico, los cuales dan lugar al conocido problema de la causa común (o screen off) y al problema de la falta de correlación. Por lo pronto, dejamos ambos asuntos a un lado en la medida en que su análisis y la amplia literatura 
conocimiento acerca de la manifestación cabal de una capacidad en un ambiente de estímulo óptimo. La individuación de una capacidad puede ser corregida mediante la elaboración de experimentos controlados que nos permiten manipular los fenómenos que poseen las capacidades relevantes que se desea investigar en un ambiente adecuado para la manifestación de sus poderes causales.

Entre los beneficios de la elaboración de diseños experimentales controlados para el estudio de las capacidades encontramos los siguientes. Primero, es posible corroborar o rechazar la existencia de capacidades que han sido postuladas hipotéticamente en la investigación científica. La fuerza de la corroboración o rechazo descansa en la confiabilidad epistémica de los resultados de una serie de manipulaciones del poder causal en cuestión. Segundo, la experimentación controlada permite corregir y ampliar la definición de una capacidad determinada en ambientes de estímulo específicos, facilitando el reconocimiento de factores ambientales óptimos para la manifestación de su poder causal y la medición de su manifestación en diversos ambientes. Y tercero, la articulación y posterior manipulación de estos experimentos genera una serie de conocimientos prácticos que propician la intervención científica en el mundo, puesto que se comprende qué elementos se han de manipular para obtener los resultados esperados, así como en qué circunstancias tiene lugar el efecto esperado.

\section{Leyes fenomenológicas: modalidad física e invariancia}

Recuérdese que nuestra hipótesis de trabajo consiste en que el disposicionalismo experimental esclarece la relación entre capacidades y leyes fenomenológicas. En esta sección damos un paso más en esta dirección, examinando la modalidad e invariancia de las leyes fenomenológicas que describen regularidades que tienen (han tenido o podrían tener) lugar en las interacciones entre capacidades.

Diversos tipos de enunciados pueden describir interacciones entre capacidades en escenarios específicos. La sola descripción de tales interacciones no le otorga estatuto nomológico a un enunciado. Por ejemplo, cuando describimos la interacción entre las hojas del té y el agua caliente en una taza, en donde el estado termodinámico del agua ocasiona reacciones moleculares en la teína de las hojas, entre otras de sus propiedades, no enunciamos una ley fenomenológica. Tampoco lo hacemos al describir la interacción que tiene lugar entre la pelota y el vidrio de la ventana cuando el desplazamiento de la primera quiebra el segundo. En breve, las leyes fenomenológicas no obtienen su estatuto nomológico a partir de la mera descripción de interacciones entre capacidades en fenómenos específicos. Igualmente, como se observó en la sección 2, el objetivo de la ciencia no consiste en articular enunciados que ofrezcan un mero catálogo de interacciones que tienen lugar en tales o cuales escenarios, vale decir, meros enunciados inductivos que nos informen acerca de lo que es el caso en tal o cual escenario físico.

a la que han dado lugar escapan al objetivo del presente trabajo. Para una exposición general de respuestas posibles a ambos problemas, véase Reichenbach (1971) y Cartwright (1983). 
¿Qué le otorga, entones, estatuto nomológico a enunciados de leyes fenomenológicas que describen la interacción entre capacidades? Sostenemos que su estatuto nomológico se fundamenta en el carácter modal de las capacidades ${ }^{3}$. Las leyes fenomenológicas expresan generalizaciones físicas acerca de la conducta de fenómenos dadas las capacidades que los constituyen. Como tales, ellas no dan cuenta de todas las capacidades que constituyen a un fenómeno, sino que ofrecen generalizaciones físicas acerca de aquellos arreglos de capacidades que resultan relevantes desde una perspectiva científica, teniendo en cuenta las hipótesis que se quiere investigar, los instrumentos de experimentación disponibles y otras consideraciones pragmáticas relevantes para dominios particulares de investigación. Además, la relevancia de las regularidades descritas por leyes fenomenológicas requiere que la interacción entre capacidades posea saliencia y estabilidad, haciéndolas accesibles a instrumentos de experimentación en diversos momentos y contextos.

Una ley fenomenológica describe, generalmente en términos de estructuras matemáticas (véase la sección 6), un espacio modal dentro del cual puede tener lugar un rango de fenómenos. Sostenemos, con ello, que las leyes fenomenológicas en cuestión funcionan como reglas de inferencia que nos permiten construir diversas clases de ambientes experimentales, modelos y simulaciones computacionales, según sea necesario atendiendo a las peculiaridades del dominio de investigación, sea que se quiera representar, explicar o predecir fenómenos.

Ahora bien, la modalidad de las leyes fenomenológicas no es meramente matemática o de dicto, sino que eminentemente de re. Vale decir, ellas describen la estructura modal de diversos arreglos de capacidades en fenómenos. La fuente de modalidad para las leyes fenomenológicas está dada por las capacidades que constituyen los diversos sistemas físicos que conforman la realidad. Tales arreglos de capacidades sustentan asimétricamente la modalidad descrita por enunciados de leyes fenomenológicas, en donde estas últimas ofrecen generalizaciones físicas acerca de distintos patrones estructurales a los que un conjunto de capacidades puede dar lugar.

Refirámonos brevemente a la invariancia de las leyes fenomenológicas. Las interacciones entre capacidades descritas por las leyes fenomenológicas tienen grados de invariancia. Al introducir modificaciones en las condiciones de estímulo de un conjunto de capacidades, se obtienen modificaciones en el arreglo estructural de la interacción entre estas propiedades. Sin embargo, los cambios que tienen lugar dependen de la robustez disposicional del arreglo de capacidades, la cual le permite a un conjunto de capacidades conservar el arreglo estructural de un sistema físico en diversos ambientes. Por supuesto, las regularidades más robustas ostentan un mayor grado de invariancia, si se las compara con arreglos de capacidades altamente sensibles al ambiente. Las regularidades tienen, en consecuencia, grados; $y$, en general, las regularidades robustas

Al hablar del fundamento del carácter modal de las leyes fenomenológicas pensamos en la expresión inglesa grounding, vale decir: la modalidad de las capacidades es el grounding en el que descansa el carácter modal nomológico de los enunciados de leyes fenomenológicas. 
son aquellas que convocan la atención de la investigación científica. Si bien las leyes fenomenológicas son válidas solo en circunstancias restringidas por condiciones ceteris paribus, los fenómenos descritos por tales leyes acarrean sus poderes causales de un contexto a otro, de modo tal que ellos tienden a comportarse de ciertas maneras más bien que de otras en diversos escenarios experimentales.

Aun cuando la invariancia de las leyes fenomenológicas se obtiene generalmente en ambientes experimentales, se observa que algunos arreglos disposicionales poseen robustez natural. Ejemplo de esto último es la relación gravitacional entre los planetas, sus lunas y el sol en nuestra galaxia. Si se considera que la masa aproximada de la tierra es $6 \cdot 10^{24} \mathrm{~kg}$ y que la masa del sol es $2 \cdot 10^{30} \mathrm{~kg}$, dada una distancia de $1.5 \cdot 10^{11} \mathrm{~m}$ y un valor para la constante $\mathrm{G}$ de $6.7 \cdot 10^{-11}\left(\mathrm{~N} \cdot \mathrm{m}^{2} / \mathrm{kg}^{2}\right)$, resulta que la atracción gravitacional aproximada de la tierra respecto del sol es:

$$
6.674 \cdot 10^{-11} \cdot 6 \cdot 10^{24} \cdot 2 \cdot\left(10^{30} / 2.25 \cdot 10^{22}\right)=3.6 \cdot 10^{22}(\mathrm{~N})
$$

Podría sostenerse, sin embargo, que no son muchos los casos de invariancia natural; en cambio, la investigación científica necesita recurrir rutinariamente a diseños de ambientes experimentales controlados que garanticen la invariancia de ciertos fenómenos. Esto se hace particularmente necesario en casos en los cuales no tenemos acceso observacional a los fenómenos en cuestión; o en donde se quiere tener acceso a la manifestación de una capacidad específica en un determinado fenómeno; o casos en los que la capacidad que se quiere investigar no se manifiesta de manera natural en nuestros ambientes, sino solo a escalas energéticas e informacionales que se obtienen en ambientes de laboratorio, tales como el caso del decaimiento de partículas y la detección de excitaciones del campo de Higgs.

Una moraleja de nuestro análisis consiste en que la invariancia de las leyes fenomenológicas se obtiene al costo de introducir condiciones ceteris paribus que explicitan requerimientos que se tienen que dar en un ambiente experimental para que se obtenga la interacción entre capacidades que satisface la descripción ofrecida por una ley fenomenológica. El disposicionalismo experimental no pasa por alto este punto en su interpretación de las leyes fenomenológicas, sobre todo considerando que sus condiciones ceteris paribus son las mismas que guían el diseño y construcción de ambientes experimentales que garantizan la manifestación de ciertas capacidades.

La sensibilidad ambiental de las capacidades impone una restricción epistémica a nuestra concepción de las leyes fenomenológicas, en tanto que la regularidad de las últimas depende asimétricamente de la regularidad de la interacción entre las primeras. Aun cuando se haya individuado una capacidad, cabe siempre la posibilidad de que ella no se manifieste de la manera esperada si los factores ambientales varían. Vale decir, un sistema físico que posee un conjunto de capacidades determinadas (más otras capacidades que podrían resultarnos inaccesibles en un momento determinado o que no son de interés para las mediciones y detecciones en un contexto experimental) podría comportarse de modo diferente al esperado si el ambiente de estímulo varía. 


\section{Generalización física y extrapolación}

El alcance de las generalizaciones físicas expresadas por leyes fenomenológicas es limitado. Si bien las leyes fenomenológicas ostentan el beneficio teórico de ofrecernos descripciones de fenómenos (a diferencia de las leyes fundamentales mencionadas en la sección 1), tales descripciones son factibles en la medida en que restringimos el alcance de la generalización física. Se manipula la situación de tal manera que la generalización física expresada por una ley fenomenológica refiera a fenómenos que, primero, posean un arreglo de capacidades específicas y, segundo, se encuentren en un ambiente experimental que conste de condiciones adecuadas. Por ello, no resulta sorprendente que un enunciado de ley fenomenológica pueda ser artificialmente falseado si se lo aplica a ambientes no controlados.

El carácter modal de un enunciado de ley fenomenológica, cuyo fundamento es la modalidad inherente de las capacidades, permite llevar a cabo extrapolaciones de diversa índole en la práctica científica. La modalidad permite que los resultados que se obtienen por medio de la experimentación con capacidades sean generalizables a las entidades que poseen las mismas capacidades en escenarios físicos similares. Esto nos permite dar cuenta de la extrapolación de conocimiento en ciencias, la cual tiene lugar de diversas maneras. Podemos extrapolar resultados desde un diseño experimental controlado a otro. Pero también podemos extrapolar enunciados de leyes fenomenológicas desde experimentos controlados hacia escenarios físicos naturales no controlados, aunque corriendo el riesgo eminente de que capacidades adicionales rompan la relación causal esperada. Puede todavía haber otros escenarios, como el de la extrapolación en términos de replicación de fenómenos desde la naturaleza hacia el diseño experimental en el laboratorio.

El rol del diseño de ambientes experimentales es fundamental. Las regularidades obtenidas en la interacción entre capacidades solamente pueden replicarse con seguridad en ambientes que proveen las condiciones de estímulo adecuadas. Además de permitir individuar capacidades, el diseño de ambientes experimentales permite proveer los factores ambientales que aseguren que tenga lugar una determinada interacción entre un conjunto de capacidades, satisfaciendo la descripción ofrecida por una ley fenomenológica.

La extrapolación de una generalización física puede permanecer invariante en diversos ambientes en la medida en que ella depende de la modalidad física de las capacidades. Igualmente, la interacción entre capacidades es más o menos robusta dependiendo de la configuración de ambientes específicos de estímulo. Aunque las capacidades se comporten de modos semejantes en ambientes semejantes, puede afirmarse también que ciertos arreglos de capacidades poseen mayor o menor robustez, siendo resistente a diversos cambios en los factores ambientales.

En breve, la invariancia de los enunciados de leyes fenomenológicas descansa en dos elementos: primero, las condiciones iniciales ceteris paribus del ambiente; y segundo, la robustez disposicional de los arreglos de capacidades. El cumplimiento de ambos elementos permite que las leyes fenomenológicas ofrezcan generalizaciones físicas robustas que pueden ser extrapoladas a otros ambientes en los que tenemos 
razones para creer que se obtienen tanto las condiciones ceteris paribus como el arreglo de capacidades.

El disposicionalismo experimental que proponemos para construir la relación entre capacidades y leyes fenomenológicas tiene el beneficio de dar cuenta de diversos aspectos de la práctica científica. Tal como se argumentó en vistas de las capacidades (sección 2), las leyes fenomenológicas no nos presentan un mero conjunto de afirmaciones iterativas acerca de lo que es el caso, sino que ellas nos informan también acerca de un espacio de modalidad física que es crucial para la articulación de descripciones, explicaciones y predicciones. El carácter experimental de las ciencias salta a la vista, por lo demás, no solamente en la investigación de sistemas físicos que encontramos dados en la naturaleza, sino también en el diseño de intrincados ambientes experimentales que nos permiten acceder a ciertas capacidades y a la confirmación de teorías y leyes, si es el caso.

La concepción experimentalista de las capacidades y de las leyes fenomenológicas se ajusta al carácter falible de la investigación científica. Nuestra primera individuación e identificación de ciertas capacidades, con los poderes causales que creemos que ellas acarrean, puede ser errónea si, por ejemplo, hemos omitido la consideración de factores que afectan la conducta del sistema físico que se investiga y que no han sido incluidos en la determinación de condiciones iniciales ceteris paribus ni en el enunciado de la ley. El asunto se torna particularmente interesante para el experimentalista en ciencias: las capacidades son sensibles al ambiente; sus manifestaciones pueden ser graduales; ellas pueden estar presentes en un fenómeno aun cuando no se manifiesten; y su interacción con otras capacidades puede ser robusta, soportando el cambio de factores ambientales e incluso el traslado desde ambientes experimentales artificiales a ambientes naturales.

Una ley fenomenológica puede ofrecernos información indirecta acerca de cómo podrían comportarse ciertos fenómenos dados en circunstancias diferentes. Examinaremos a continuación el caso de la ley de Fresnel (ver sección 6). Tal enunciado de ley no solamente describe cómo se comporta un sistema físico bajo determinadas condiciones iniciales, sino que también entrega información para anticipar cómo se comportaría en condiciones iniciales diferentes. Esto es posible dadas las características de las capacidades, a saber, si se conoce cuál es la variación en la manifestación asociada con factores ambientales, entonces es posible generar anticipaciones de diverso tipo acerca de lo que podría ser el caso. El alcance de las anticipaciones dependerá de factores tales como el conocimiento y control que se tenga de las capacidades que componen los distintos sistemas y sus ambientes, la posibilidad de una manifestación óptima, e información experimental adicional que facilite la construcción de modelos que consideren las condiciones iniciales relevantes. Volveremos sobre estos puntos en la sección siguiente.

\section{El caso de la ley de Fresnel}

El caso de estudio de la ley de reflexión y refracción de ondas de luz de Fresnel ilustra las ideas principales de nuestro análisis del disposicionalismo experimental y sus 
consecuencias para las leyes fenomenológicas que hemos defendido. Fresnel contribuyó a la articulación de teorías acerca de la propagación, polarización, reflexión y refracción de la luz. Su trabajo ayudó a que científicos franceses e ingleses, que inicialmente defendían la teoría corpuscular de la luz, se convencieran de los beneficios teóricos de la teoría ondulatoria de Fresnel. En efecto, a diferencia del corpuscularismo, la teoría ondulatoria daba cuenta de una variedad de fenómenos ópticos de manera cuantitativamente unificada (Darrigol 2012, p. 224).

Restringiremos nuestro análisis a la llamada ley de Fresnel (LF) concerniente a la refracción y reflexión de ondas de luz polarizadas. Tal ley óptica describe el comportamiento de la luz polarizada en términos ondulares al interactuar con la interfaz entre dos medios, permitiendo calcular la intensidad de las fracciones de onda que se reflejan y se refractan en la interfaz, transmitiéndose por el segundo medio. La ecuación que enuncia LF expresa la intensidad luminífera mediante la combinación de información experimental, la ley de Snell, la ley de refracción y la ley de conservación de la energía:

$$
i_{R}=-\frac{\sin \left(\theta_{i}\right) \cos \left(\theta_{i}\right)-\sin \left(\theta_{T}\right) \cos \left(\theta_{T}\right)}{\sin \left(\theta_{i}\right) \cos \left(\theta_{i}\right)+\sin \left(\theta_{T}\right) \cos \left(\theta_{T}\right)} .
$$

Según LF, cuando un rayo incidente de luz polarizada interactúa con la interfaz entre dos medios ópticos lineares con ángulo $\theta_{i}$, se refleja en el medio de incidencia un haz de luz correspondiente a una fracción de onda con ángulo $\theta_{r}$, y se refracta otro haz de luz correspondiente a la fracción de onda restante que se propaga por el medio de transmisión con un ángulo $\theta_{t}$ La Figura 1 muestra la manera en que LF concibe la relación entre estos factores y la intensidad de las ondas:

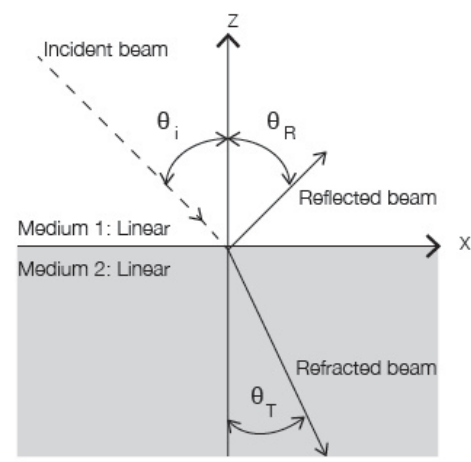

Figura 1. (Wright 2017)

4 Para un estudio histórico detallado del presente caso de estudio, véase Darrigol 2012, capítulo 5. Véase también Wright 2017. 
Este caso óptico refleja las características de las leyes fenomenológicas que hemos defendido en las secciones precedentes. Primero, de acuerdo a la distinción ofrecida en la sección 1, esta ley es fenomenológica, puesto que describe las manifestaciones macroscópicas explicadas por la dispersión ocurrente a un nivel subatómico (Hecht 2002, p. 86). Segundo, los fenómenos descritos por esta ley solo tienen lugar si se obtienen ciertas condiciones ambientales. En efecto, para que el fenómeno descrito por LF ocurra "tiene que haber una interfaz entre dos medios para que el haz de luz se refleje y refracte” (Wright 2017,p. 46). Así, el fenómeno de la refracción y reflexión de ondas de luz está constituido por un arreglo de capacidades que precisa de condiciones de estímulo (sección 2).

En tercer lugar, podemos identificar ambientes de estímulo óptimos y ambientes que, en cambio, no son tales. LF se aplica con precisión a circunstancias en las cuales los dos medios son isotrópicos o lineares ${ }^{5}$. La linearidad (o isotropía) entre los medios es una condición de estímulo óptimo. Entre las condiciones óptimas y las que no lo son se evidencia un continuum. La linearidad puede romperse gradualmente si varía el índice de refracción de alguno de los dos medios ${ }^{6}$. Por lo demás, los cambios graduales en el ambiente se corresponden con cambios graduales en la manifestación. En nuestro caso, la reflexión variará conforme a la diferencia entre los índices de refracción de los medios. Mientras más gradual sea la transición entre estos, menor será la reflexión. Por el contrario, mientras más abrupta sea la transición entre estos, mayor será la reflexión. Hecht pone de relieve este punto:

Si la transición entre dos medios es gradual -vale decir, si la constante dieléctrica (o el índice de refracción) cambia desde un medio a otro a lo largo de la distancia entre una onda o más-, habrá muy poca reflexión; la interface efectivamente se desvanece. Por otro lado, una transición desde un medio a otro a una distancia de $1 / 4$ o menos se comporta de manera muy parecida a un cambio totalmente discontinuo (Hecht 2002, p. 95).

Los cambios en el índice de refracción no solo alteran el haz de luz reflejado. Si el medio de transmisión no es linear con el medio de incidencia, "se generan diferencias cuantitativas y cualitativas" (Wright 2017, p. 47). En efecto, en tales circunstancias se genera un segundo haz de luz en el medio de transmisión, llamado segunda onda armónica.

Una cuarta consideración es la siguiente: LF es una ley fenomenológica ceteris paribus (sección 4). LF se aplica a ondas de luz en circunstancias físicas particulares.

5 Un material es isotrópico o linear cuando el orden atómico es lineal de modo tal que no se presentan direcciones que difieren entre sí. Cuando se miden las propiedades de estos materiales el resultado es invariante sin importar la dirección en la que se efectúa la medición.

$6 \quad$ Al hablar del fundamento del carácter modal de las leyes fenomenológicas pensamos en la expresión inglesa grounding, vale decir: la modalidad de las capacidades es el grounding en el que descansa el carácter modal nomológico de los enunciados de leyes fenomenológicas. 
El fenómeno descrito por la ley solo tiene lugar si se cumplen las condiciones óptimas. Por cierto, en nuestro ejemplo solamente hemos abordado algunas de las circunstancias relevantes. No obstante, la polarización del haz de luz y la intensidad son factores que han de permanecer estables. LF permanecerá invariante en todo sistema físico que instancie las condiciones ceteris paribus, lo cual da cuenta de cierta robustez disposicional, pero también del alcance restringido de la generalización física y de la extrapolación (sección 5).

En consecuencia, LF describe el comportamiento ondular del haz de luz en circunstancias óptimas en las que los medios son lineares. Igualmente, LF permite predecir cómo se comportará el haz de luz en circunstancias que no son óptimas, cumpliendo un rol inferencial en la articulación de extrapolaciones y predicciones. Ello evidencia el carácter modal de LF, que sirve de guía para explorar nuevos escenarios de modalidad física.

\section{Consideraciones finales}

En este artículo hemos investigado la relación entre capacidades y leyes fenomenológicas desde la perspectiva de un disposicionalismo experimental que se distancia del categoricalismo y del disposicionalismo metafísico estándar en el debate acerca de propiedades. Aunque nuestra concepción se encuentra, por supuesto, más cerca a la segunda postura, hemos sostenido que las capacidades no están asociadas analíticamente a la manifestación de un único poder causal. En cambio, cada capacidad puede dar lugar a un rango de comportamientos cuya manifestación depende del ambiente en el que se encuentran. El análisis de las manifestaciones de las capacidades requiere, por ende, la introducción de consideraciones probabilísticas que establecen correlaciones entre una capacidad y su posible efecto en determinadas circunstancias.

El disposicionalismo experimental esclarece, igualmente, diversos aspectos de las leyes fenomenológicas. Tales leyes, hemos argumentado, tienen un carácter modal, informándonos acerca de lo que podría ser el caso en circunstancias a las que no tenemos acceso en un momento determinado. La modalidad otorga un estatuto nomológico a las leyes, distinguiéndolas de otra clase de enunciados. Ahora bien, la modalidad física, acerca de la cual nos informan profusamente nuestras teorías científicas y en particular los enunciados de ley, radica inherentemente en las capacidades. Vale decir, si las teorías y las leyes en ciencias tienen un carácter modal es porque ellas nos informan acerca de un rango de comportamientos físicamente posibles.

Un desafío pendiente dice relación con la posibilidad de extender nuestro análisis experimentalista a las leyes fundamentales. ¿Obtienen las leyes fundamentales su modalidad a partir de capacidades? ¿Requieren los enunciados de leyes fundamentales condiciones ceteris paribus? ¿Se restringe el alcance de la generalización física expresada por enunciados de leyes fundamentales a ambientes experimentales restringidos natural o artificialmente? Tales cuestiones quedan pendientes. Nuestro análisis se ha limitado al caso de las leyes fenomenológicas. Morrison (2015, capítulo 1) ha cuestionado el argumento de Cartwright (1999) en contra de las leyes fundamentales, elaborando 
estrategias para derivar verdades empíricas a partir de leyes fundamentales en su análisis del rol de las matemáticas en la construcción de modelos y simulaciones computacionales acerca de sistemas físicos a los que nos tenemos acceso epistémico directo.

En el presente trabajo, hemos preferido decir poco acerca del estado actual del debate en filosofía de las leyes de la naturaleza, en donde las propuestas se clasifican, generalmente, en humeanas, realistas y eliminativistas. El desafío, en este caso, consistiría en indicar con precisión qué concepción de ley adoptamos en nuestro análisis. Permítasenos introducir una observación breve al respecto. Ni el eliminativismo ni el realismo se acomodan a nuestra propuesta. Por cierto, nuestra concepción no es eliminativista acerca de las leyes en la práctica científica, puesto que sostenemos que el concepto de ley juega un rol clave en la práctica científica. Nuestra concepción de las leyes fenomenológicas igualmente se aleja de las propuestas metafísicas usuales acerca de leyes de la naturaleza, a saber: en lugar de reificar las leyes postulando una clase especial de hechos metafísicos, hemos preferido hablar de enunciados de leyes fenomenológicas cuyo estatuto nomológico se funda en la modalidad física de las capacidades.

En consecuencia, la opción que al parecer nos queda disponible, si nos atenemos a la clasificación estándar del estado del arte sobre leyes de la naturaleza, es el humeanismo. Con el humeanismo, de hecho, compartimos la idea de que las leyes son enunciados; sin embargo, contra el humeanismo sostenemos que las leyes nos informan acerca de un espacio modal de posibilidades físicas. El defensor del humeanismo carece de la modalidad física que, según el disposicionalismo experimental, es inherente a las capacidades y que resulta crucial para las leyes fenomenológicas. Las regularidades humeanas son subjetivas, mientras que las regularidades expresadas por leyes fenomenológicas, según el disposicionalismo experimental, son objetivas, dependiendo asimétricamente de las capacidades que constituyen los fenómenos. El disposicionalismo experimental concibe un mundo físico pleno de capacidades, a las que accedemos de manera falible y corregible por medio de la experimentación y del análisis probabilístico; y sostenemos que las leyes fenomenológicas que describen algunos aspectos de este mundo nos ofrecen descripciones restrictas acerca de relaciones causales que aceptan matices y grados, dependiendo del ambiente de estímulo en el que se instancien los diversos arreglos de capacidades.

\section{Referencias bibliográficas}

Cartwright, Nancy (1983), How de Laws of Physics Lie. Oxford: Clarendon Press. (1989), Nature's Capacities and their Measurement. Oxford: Clarendon Press.

(1999), The dappled World: A Study of the Boundaries of Science. Cambridge: Cambridge University Press.

(2002), "Reply", Book Symposium on The Dappled World, Philosophical Books 43: 271-278. 
(2007), Causal Powers: What are they? Why we need them? What can be done with them and what cannot? Technical Report 04/07, Center for Philosophy of Natural and Social Science.

Chakravartty, Anjan (2007), "Causal Realism and Causal Processes", A Metaphysics for Scientific Realism. New York: Cambridge University Press; pp. 89-119.

(2017), Scientific Ontology: Integrating Naturalized Metaphysics and Voluntarist Epistemology. New York: Cambridge University Press.

Darrigol, Olivier (2012), "Interference, Polarization, and Waves in the Early Nineteenth Century", A History of Optics. Oxford: Oxford University Press; pp. 166-124.

Dupré, John (1984), "Probabilistic Causality Emancipated", Midwest Studies in Philosophy IX: 169-175.

Hecht, Eugene (2002), “The Propagation of Light”, Optics. San Francisco: Addison Wesley; pp. 86-148.

Morrison, Margarete (2015), Reconstructing Reality: Models, Mathematics and Simulations. Oxford: Oxford University Press.

Mosterín, Jesús y Torretti, Roberto (2002), Diccionario de Lógica y Filosofía de la Ciencia. Madrid: Alianza Editorial.

Mumford, Stephen (1998), "Dispositional-Categorical Distinction”, Dispositions. New York: Oxford University Press; pp. 64-92.

(2009), "Causal Powers and Capacities", en H. Beebee, C. Hitchcock, P. Menzies, eds., The Oxford Handbook of Causation. Oxford: Oxford University Press; pp. 242-253.

Psillos, Stathis (2008), “Cartwright's Realist Toil: From Entities to Capacities”, en S. Hartmann, C. Hoefer, L. Bovens, eds., Nancy Cartwright's Philosophy of Science. New York: Routledge; pp. 167-195.

Reichenbach, Hans (1971), The Direction of Time. Berkeley: California University Press.

Wright, Aaron (2017), "Fresnel's Law, ceteris paribus", Studies in History and Philosophy of Science 64: 38-52. 